\title{
Population Differentiation Within Anisogramma anomala in North America
}

\author{
Megan F. Muehlbauer, ${ }^{1, \dagger}$ Janine Tobia, ${ }^{2}$ Joshua A. Honig, ${ }^{2}$ Ning Zhang, ${ }^{2}$ Bradley I. Hillman, ${ }^{2}$ \\ Kaitlin Morey Gold, ${ }^{3}$ and Thomas J. Molnar ${ }^{2}$ \\ ${ }^{1}$ Rutgers University Cooperative Extension of Hunterdon County, Flemington, NJ 08822-2900; ${ }^{2}$ Department of Plant Biology, Rutgers \\ University, New Brunswick, NJ 08901; and ${ }^{3}$ Department of Plant Pathology, University of Wisconsin, Madison, WI 53706-1598 \\ Accepted for publication 9 December 2018.
}

\begin{abstract}
Anisogramma anomala, a biotrophic ascomycete in the order Diaporthales, causes eastern filbert blight (EFB) of hazelnuts (Corylus spp.). Until recently, little has been documented on its genetic diversity and population structure. In this study, 18 simple sequence repeat markers were used to fingerprint 182 accessions of the fungus originating from across North America. Our results, based on summary statistics of the allelic data, a discriminant analysis of principal components (DAPC) scatterplot, an unweighted pair group method with arithmetic mean (UPGMA) dendrogram, and analysis of multilocus genotypes, show that A. anomala exhibits considerable genetic diversity across multiple populations. Eleven clusters were resolved from the DAPC scatterplot,

five of which were validated by statistically supported clusters in the UPGMA dendrogram. The 11 DAPC clusters were statistically significant via an analysis of molecular variance. Dendrogram topology and DAPC scatterplot groups showed some correlation with collection origin; samples collected in proximity tended to cluster together and be genetically similar. However, some locations held populations that were diverse and some populations with a high degree of similarity had disparate origins, suggesting movement by humans. Overall, the results demonstrate the presence of multiple, genetically distinct populations of A. anomala in North America and serve as a reference to assist in understanding and managing EFB.
\end{abstract}

Anisogramma anomala (Peck) E. Müller is the causal agent of the disease eastern filbert blight (EFB) of hazelnuts (Corylus spp.). It is an obligate, biotrophic ascomycete in the order Diaporthales and is native to a wide expanse of eastern North America where it is found associated with its native host, the wild American hazelnut (C. americana Marshall) (Gottwald and Cameron 1979, 1980a; Pinkerton et al. 1995). Although the fungus causes little damage to C. americana, it causes large perennial stem cankers, branch dieback, and ultimately death of most cultivars of the commercially important European hazelnut (C. avellana L.) (Capik and Molnar 2012; Fuller 1908; Johnson and Pinkerton 2002; Pinkerton et al. 1993; Weschcke 1954). EFB is considered to be the primary limiting factor of hazelnut production in eastern North America (Thompson et al. 1996) and its management causes significant expense in Oregon, where $99 \%$ of the U.S. commercial crop is grown (Julian et al. 2009). To date, the fungus has not been found outside of North America (European and Mediterranean Plant Protection Organization 2012).

The first descriptions and reports of the EFB pathogen were made in the northeastern United States in the late 1800s to early 1900s (Ellis and Everhart 1892 [page 793]; Fuller 1908; Peck 1874). It was recognized as a serious threat to the burgeoning hazelnut industry in the Pacific Northwest where the fungus was nonexistent, and a strict quarantine was established on the movement of Corylus plants west of the Rocky Mountains to prevent its spread (Barss 1921,

†Corresponding author: M. F. Muehlbauer; muehlbauer@njaes.rutgers.edu

Funding: Funding for this research was provided by the New Jersey Agricultural Experiment Station, the Rutgers Center for Turfgrass Science, and the U.S. Department of Agriculture National Institute of Food and Agriculture (Hatch funds and Specialty Crops Research Initiative Competitive Grants 2016-51181-25412 and 2009-51181-06028).

*The $\boldsymbol{e}$-Xtra logo stands for "electronic extra" and indicates that two supplementary tables and one supplementary data set are published online.

The author(s) declare no conflict of interest.

C 2019 The American Phytopathological Society
1930; Pinkerton et al. 1992). Despite the quarantine, EFB was introduced into southwest Washington in the 1960s (Davison and Davidson 1973), where it devastated local production. Today, EFB has spread south and is present across the entire Willamette Valley, where it imparts a significant economic impact on $C$. avellana production (Julian et al. 2009; Pscheidt et al. 2014).

A. anomala is reported to be homothallic and reproduces only by ascospores (Gottwald and Cameron 1979). In the Pacific Northwest, where most of the research on the pathogen's biology has been completed, ascospores are released from November to May (Gottwald and Cameron 1980b). Liberated ascospores are then moved by rain splash and wind-driven rain (Pinkerton et al. 1998). Because of the high costs associated with chemical-based control, the development and utilization of resistant cultivars is considered to be the best EFB management strategy (Julian et al. 2009).

In support of this approach, considerable research has been done over the past 2 decades to identify sources of resistance for use in breeding (Mehlenbacher 1994; Molnar et al. 2005). The first source of resistance identified was 'Gasaway', a late-shedding pollinizer from Washington found to confer resistance through a dominant allele in the heterozygous state at a single locus (Mehlenbacher et al. 1991, 2004). Despite the poor nut production traits of Gasaway, breeders at Oregon State University (OSU) in Corvallis, Oregon, have used it to develop a number of EFB-resistant, commercial-quality cultivars (Mehlenbacher et al. 2009, 2011, 2013,2014 ), which have provided the basis for the expansion of Oregon's hazelnut industry by $\sim 6,000$ ha over the past 5 years (S. Mehlenbacher, personal communication).

Concerns over the long-term durability of the Gasaway $R$-gene motivated researchers to find additional sources of resistance. Over the past 15 years, many hundreds of cultivars and thousands of seedling accessions of $C$. avellana have been screened, resulting in the identification of additional EFB-resistant plants (Capik et al. 2013; Chen et al. 2005, 2007; Colburn et al. 2015; Coyne et al. 1998; Leadbetter et al. 2015, 2016; Lunde et al. 2000; Molnar et al. 2007; Muehlbauer et al. 2014; Sathuvalli et al. 2009, 2010, 2011a, b). However, several studies have suggested that pathogenic variation exists in A. anomala. Osterbauer (1996) found that clonal plants 
related to Gasaway developed small, sunken lesions (although lacking stromata) when challenged with $A$. anomala from Minnesota and Ontario but developed no signs or symptoms of the disease when challenged with the fungus collected from Oregon and four other regions. Later, Molnar et al. (2010b) showed that trees of Gasaway developed EFB only when exposed to A. anomala from Michigan. In the field, a differential response to EFB has also been observed (Molnar et al. 2010a). Broadly, the results of greenhouse studies and field observations suggest that genetic variation likely exists within the pathogen.

In the first attempt to study molecular genetic variation of A. anomala, Osterbauer (1996) sequenced and compared the internal transcribed spacer regions of 67 accessions from five locations in Oregon and Washington but found little sequence divergence among them. She also examined 33 accessions collected from multiple locations across North America with random amplified polymorphic DNA markers. The results of her work showed an apparent genetic profile similarity between accessions from Oregon, Washington, New York, and Ontario, Canada.

$A$. anomala has been sequenced and simple sequence repeat (SSR) markers have been identified (Cai et al. 2013). Results from the amplification of $30 \mathrm{~A}$. anomala samples showed that the samples were distinct and resolved two major groups: one consisting almost entirely of samples collected from New Jersey, and the other holding those collected from the Great Lakes region and Oregon (Cai et al. 2013). The preliminary analysis by Cai et al. (2013) confirmed that the fungus is genetically diverse and laid the framework for a more indepth study. The objective of the current study is to expand upon the work of Cai et al. (2013), using 18 SSR markers to investigate the genetic diversity and population structure of 182 samples of A. anomala collected from 43 locations across North America.

\section{MATERIALS AND METHODS}

A. anomala samples and DNA extractions. Infected hazelnut stems containing mature A. anomala stromata were collected between January 2011 and March 2013 from hazelnut trees growing at 43 different locations spanning 12 states and one Canadian Province. A total of 182 samples were obtained and examined in this study (Supplementary Table S1). A majority of the samples were collected from the cultivated hazelnut $C$. avellana $(n=105)$; however, 15 were collected from $C$. americana, 18 from known hybrids of C. americana $\times$ C. avellana, one from C. colurna, 21 from plants whose origin spans hybrids of $C$. americana $\times$ C. avellana $\times$ C. colurna, and 22 whose Corylus species identity was unknown. When possible, the infected stems were collected from different trees at each location to obtain samples derived from separate infection events. If only one infected tree was available, stems were collected from far apart in the canopy in an attempt to avoid collecting cankers from the same infection event (and potentially the same genotype). Stems were placed in polyethylene bags and stored at $-20^{\circ} \mathrm{C}$ until use. Although the EFB canker on an individual stem contained multiple stromata, ascospores were obtained from the perithecial content of a single stroma for DNA extractions.

To maintain consistency between studies, DNA of the A. anomala genotypes used for SSR primer testing and selection (described in the subsequent section) was the same as that used by Cai et al. (2013). The fungal samples were obtained from New Jersey (NJ_Cream_ Ridge_6), Ohio (OH_Newark_11), Oregon (OR_Corvallis_1), and Wisconsin (WI_Moquah_1) and were chosen to represent geographically distant regions of the United States. For these samples, ascospores were germinated and grown in axenic culture (Stone et al. 1994) to obtain DNA for primer testing. DNA was extracted using a DNAeasy plant kit (Qiagen, Germantown, MD) (Cai et al. 2013).

DNA extractions of the remaining 178 samples were modified from those described in Cai et al. (2013). Instead of culturing the ascospores, the perithecial matrix from a single stroma was obtained and aseptically transferred to a $1.5-\mathrm{ml}$ microcentrifuge tube containing $1 \mathrm{ml}$ of sterile water. The ascospore suspension was then filtered through a $40-\mu \mathrm{m}$ nylon mesh cell strainer and centrifuged at 5,000 rpm for $2 \mathrm{~min}$. The water was removed aseptically by pipette, leaving behind concentrated pellets of spores that were stored in microcentrifuge tubes at $-80^{\circ} \mathrm{C}$. DNA was extracted from previously stored spore pellets using a modified MoBio Ultra Clean Plant DNA Isolation kit (MoBio, Carlsbad, CA).

SSR primers, amplification, and scoring. A total of 18 primers were used to genotype the 182 samples, five of which were previously described and used in Cai et al. (2013) (primers Aa00689, Aa02342, Aa16574, Aa03927, and Aa02944) (Supplementary Table $\mathrm{S} 2$ ). The remaining 13 primers were identified as a component of the current study, utilizing the SSR primer screening method described in the previous section. Briefly, 96 SSR loci were chosen from the genome-wide microsatellite database (39,361 loci) generated by Cai et al. (2013). The set included 66 dinucleotide, 27 trinucleotide, and eight tetranucleotide repeat motifs. The primers were amplified in the four "tester" accessions and examined for polymorphic alleles. Primers that resolved at least three different alleles per locus were considered targets for final selection and use in the current diversity study. It should be noted that $A$. anomala is a homothallic fungus; extractions from single stroma yielded single genotype isolations consisting of haploid ascospores, and these haploid ascospore accessions yield one allele at each locus (Cai et al. 2013). Thus, the maximum expected number of different alleles across the four "tester" genotypes per locus would be four.

The following amplification protocol was used for both primer testing and genotyping the samples. The M13 (-21) 18-bp sequence was added to the $5^{\prime}$ end of all forward primers as a cost-effective means of fluorescent labeling of polymerase chain reaction (PCR) fragments (Schuelke 2000), and the "PIG-tailing" sequence (GTTTCTT) was included at the $5^{\prime}$ end of all reverse primers to reduce uncertainty in scoring "true" versus "plus-A" alleles (Brownstein et al. 1996). Primers were synthesized by Integrated DNA Technologies (Coralville, IA). PCR genotyping reactions were performed in 96-well plates in $13-\mu$ l reaction volumes containing approximately $5 \mathrm{ng}$ of genomic DNA, 10× Ramp-Taq PCR buffer (Denville Scientific, Metuchen, $\mathrm{NJ}$ ), $2.0 \mathrm{mM}$ of $\mathrm{MgCl}_{2}$, $0.25 \mathrm{mM}$ each of dNTP (Denville Scientific), $0.5 \mathrm{U}$ of Ramp-Taq DNA polymerase (Denville Scientific), 0.5 pmol of forward primer with 5' M13 (-21) addition, 1.0 pmol of reverse primer with 5' PIGtail addition, and $1.0 \mathrm{pmol}$ of FAM, NED, PET, or VIC fluorescently labeled M13 (-21) primer. PCR cycling was performed in GeneAmp 9700 thermal cyclers (Applied Biosystems, Foster City, CA) using the following parameters: initial denaturation of $94^{\circ} \mathrm{C}$ for $5 \mathrm{~min}$; followed by 30 cycles of $94^{\circ} \mathrm{C}$ for $30 \mathrm{~s}, 55^{\circ} \mathrm{C}$ for $45 \mathrm{~s}$, and $72^{\circ} \mathrm{C}$ for $45 \mathrm{~s}$; followed by 20 cycles of $94^{\circ} \mathrm{C}$ for $30 \mathrm{~s}, 53^{\circ} \mathrm{C}$ for $45 \mathrm{~s}$, and $72^{\circ} \mathrm{C}$ for $45 \mathrm{~s}$; followed by a final extension of $72^{\circ} \mathrm{C}$ for $10 \mathrm{~min}$.

PCR products were run on a capillary electrophoresis genetic analyzer (ABI 3500xl; Applied Biosystems) and were sized using a LIZ600 size standard (Applied Biosystems). Two additional controls were added to each 96-well plate for the genotyping portion of the experiment: accession OR_Corvallis_2 from OSU, originally sequenced by Cai et al. (2013), and the GeneScan Installation Standard DS-33 (Applied Biosystems). SSR marker testing and genotyping results were analyzed using GeneMapper 4.0 (Applied Biosystems).

Data analysis. Summary statistics were calculated for each SSR primer pair using the program PowerMarker 3.25 (Liu and Muse 2005); parameters calculated included allele number per locus, frequency of the most common allele, gene diversity (expected heterozygosity, the probability that two randomly chosen alleles in a population are different), and polymorphism information content (PIC) value. Allele frequencies were generated by the same program and used to construct a genetic distance matrix. 
A discriminant analysis of principal components (DAPC) was used to determine the number of clusters the population of accessions could be assigned to, without a priori assignment of individuals to populations. The DAPC analysis was run in $\mathrm{R}$ version 3.5.1 (R Core Team 2018), using the package Adegenet (Jombart 2008; Jombart and Ahmed 2011). Clusters were found through $K$-means clustering of principal components. Subsequently, $K$ (optimal cluster number) was inferred as the number of clusters where the Bayesian information criterion shows a minimal increase or decrease.

Using Bruvo's distance, the unweighted pair group method with arithmetic mean (UPGMA) dendrogram was constructed to corroborate the clusters identified through the DAPC analysis. Bruvo's distance results in clustering of accessions based on mutation models and relative distance between alleles based on the SSR number at each locus (Kamvar et al. 2014, 2015). The UPGMA analysis was run using the R package (R Core Team 2018) poppr version 2.8.0 (Kamvar et al. 2014, 2015).

The full data set was then analyzed using the program MLGsim (Stenberg et al. 2003) to determine the number of multilocus genotypes (MLGs) present in each population. Concurrently, a simulation approach (1,000 simulations) was used in MLGsim to calculate the statistical significance of the likelihood statistic $\left(P_{\text {sex }}\right)$ that any MLGs observed more than once in a population were the result of clonal reproduction. If a $P_{\text {sex }}$ value was found to be significantly low $(P \leq 0.001)$, the corresponding MLG was considered as likely to have arisen by clonal reproduction. Using the results of this analysis, a clone-corrected data set was created; for every location, only one genotype was included for each MLG if clonal MLGs (as indicated by significantly low $P_{\text {sex }}$ values) were deemed to be present at a location. Both the full and clone-corrected data sets were then used in a subsequent analysis to determine the effect of possible clonal reproduction and homothallism on population structure and genetic diversity.

MLG assessment and analysis of linkage disequilibrium were conducted using the program MultiLocus version 1.2 (Agapow and Burt 2001). These analyses were performed to assess the degree of recombination occurring in both the individual populations and within the entire collection of accessions, using both the complete and clone-corrected data sets. The populations were analyzed for total number of MLGs, frequency of the most commonly occurring MLG, and genotypic diversity (probability that two individuals taken at random will have the same MLG). In addition, two linkage disequilibrium indices were calculated: the index of association $\left(I_{A}\right)$ and a modified version of $I_{A}\left(\bar{r}_{d}\right)$, which is different from $I_{A}$ in that it is not correlated to the number of loci amplified. Both statistics were analyzed by 1,000 permutations per population data set. Values that were significantly different from 0 suggest linkage disequilibrium or clonal reproduction in that population.

An analysis of molecular variance (AMOVA) was performed to assess whether the populations (with and without clonal genotypes) were statistically different from each other using

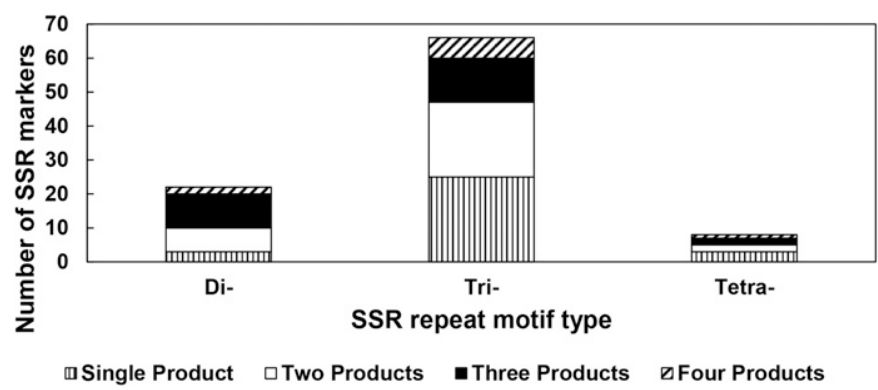

Fig. 1. Results of the screening of 96 simple sequence repeat (SSR) markers with pooled DNA from four Anisogramma anomala accessions and the number of products resolved for each repeat type. scaled estimator of differentiation $\left(\Phi_{\mathrm{PT}}\right)$ statistics in the program GenAlEx 6.502 (Peakall and Smouse 2006, 2012). Interpopulation pairwise $\Phi_{\mathrm{PT}}$ values were calculated between all of the consensus populations with statistical significance at $P \leq 0.01$. Results from the AMOVA were also utilized to determine the variance within and among populations of genotypes, as well as to determine how much variance each of the consensus populations contributed to the total within-population variance.

Spatial autocorrelation analysis was performed using the program GenAlEx 6.502 (Peakall and Smouse 2006, 2012) to examine the arrangement of genotypes over geographic space and assess whether accession genotypes can be correlated to collection sites. The degree of autocorrelation was calculated using the autocorrelation coefficient $(r)$, which is a measure of genetic similarity between pairs of accessions in a particular distance class. Distance classes were chosen such that an even number of samples were included in each class, and total geographic distance spanned 0 to $4,146 \mathrm{~km}$. The $r$ values ranged from -1 (negative spatial autocorrelation) to +1 (positive spatial autocorrelation) and the significance of $r$ values was calculated by comparing the observed value with those obtained from 999 permutations of the samples. Using the permuted correlograms, a 95\% confidence interval was constructed, and when $r$ fell above the confidence interval (positive $r$ value), significant spatial genetic structure was inferred.

\section{RESULTS}

Primer screening. Allelic data from the 96 SSR markers amplified in each of the four "tester" accessions are shown in Supplementary Data Set S1. Loci representing all three nucleotide motif types (dinucleotide, trinucleotide, or tetranucleotide repeats) resulted in polymorphic alleles with the number of SSR markers resulting in one, two, three, and four products for each repeat type (Fig. 1). Overall, 34 of the 96 total loci yielded three or four alleles each, with the dinucleotide repeats resulting in the greatest proportion of polymorphic loci. From these 34 polymorphic SSR markers, 13 were selected for use in this study, in addition to five previously characterized primers (Cai et al. 2013).

Summary statistics. Summary statistics for each of the 18 SSR markers used for the population diversity study are listed in Table 1. Major allele frequencies ranged from 0.24 to 0.62 , with a mean value of 0.41 . The number of alleles per locus ranged from 8 to 14 , with a mean of 9.5; and the gene diversity values ranged from 0.57 to 0.87 , with a mean of 0.73 . The PIC values ranged from 0.52 to 0.85 , with an average value of 0.70 .

Population structure analysis. The DAPC analysis supported $K=11$ clusters, which are visualized as a scatterplot and described as follows (Fig. 2). Population 1 primarily contains accessions from New Jersey $(n=14)$, New York $(n=3)$, and Pennsylvania $(n=2)$, with the exception of one accession from Ino, Wisconsin. Population 2 holds eight accessions, five of which were collected in Jamaica Plain, Massachusetts $(n=5)$. The remaining three accessions were obtained from New Brunswick, New Jersey.

Population 3 holds accessions 36 to 49, which form a cluster from disparate geographic origins, including four from Harrisburg, Pennsylvania, four from Leslie, Michigan, two from Boothbay, Maine, and all accessions collected from Oregon (two from Corvallis and two from Wilsonville). Population 4 holds accessions 91 to 104, nine of which originate from New Brunswick, New Jersey, along with two from Harrisburg, Pennsylvania, and three from Etters, Pennsylvania ( $<20 \mathrm{~km}$ apart). Population 5 holds accessions 66 to 72, which constitute all of the $(n=7)$ accessions in this cluster and consist of those collected from New Franklin, Missouri, and one from East Lansing, Michigan (University of Michigan).

The population 6 cluster $(n=16)$ holds accessions from a diversity of locations, including eight accessions from Hop Bottom, 
Pennsylvania, three accessions from Courtland, Ontario, and two accessions from McGraw, New York. Population 7 encompasses the largest group of 36 accessions representing a diversity of backgrounds. These accessions span collection origins of Illinois $(n=1)$, New Jersey $(n=1)$, Pennsylvania $(n=1)$, Michigan $(n=9)$, Minnesota $(n=7)$, Wisconsin $(n=14)$, and Ontario, Canada $(n=3)$. Population 8 accessions 144 to 157 comprise this entire population, all of which are derived from three cities in New Jersey (Cream Ridge, $n=1$; Adelphia, $n=2$; Rahway, $n=4$; and New Brunswick, $n=7$ ). This population is noteworthy in that it is the most distantly related population in relation to the other isolates.

The population 9 cluster holds accessions 119 to 138 . The majority of the accessions $(n=15)$ are from Jamaica Plain, Massachusetts, whereas the remaining four are from New Brunswick, New Jersey.

Population 10 holds accessions 18 to 33, which contains mostly accessions from New York and Ontario, with a few from Minnesota. It includes five accessions from Belmont, New York, and four from Angelica, New York, representing orchards located $\sim 10 \mathrm{~km}$ apart. Three accessions come from Niagara on-the-Lake, Ontario, and three more are from Toronto, Ontario $(\sim 180 \mathrm{~km}$ from the New York locations). In addition, two from Montevideo and one from Western Minnesota were placed in this group.

Population 11 holds all 11 accessions collected from Newark, Ohio (Dawes Arboretum) (accessions 106 to 116). In addition, this cluster holds one accession from each of the following disparate locations: East Lansing and Molt, Michigan; Findley Lake, New York; New Brunswick, New Jersey; and Sparta, Ontario.

Clustering analysis. Based on the UPGMA dendrogram, there is no cohesive clustering of accessions based on collection host (Fig. 3). Six accessions from three different locations (New York, Ontario, and Wisconsin), which were collected from two different host species $(C$. americana and a $C$. americana $\times C$. avellana hybrid), rooted the tree. Comparing the UPGMA results to those of the DAPC scatterplot, the UPGMA dendrogram corroborates five of the DAPC clusters. The corroborated clusters are 3, 4, 5, 8, and 10 and encompass 67 accessions, approximately a third of the accessions in this study. Another two populations of accessions, those from Newark, Ohio, and Hop Bottom, Pennsylvania, were supported in the UPGMA dendrogram, but neither comprised an entire DAPC population. The remaining accessions, about two thirds of those included in the study, support a lack of population structure.

MLG and linkage disequilibrium analysis. In total, the 182 accessions were shown to comprise 141 unique MLGs. Ten of the 11 populations held at least one pair of clonal genotypes from either the

TABLE 1. Summary statistics for each simple sequence repeat marker used in the analysis

\begin{tabular}{lcccc}
\hline Marker & $\begin{array}{c}\text { Major allele } \\
\text { frequency }\end{array}$ & $\begin{array}{c}\text { Allele } \\
\text { number }\end{array}$ & $\begin{array}{c}\text { Gene } \\
\text { diversity }\end{array}$ & $\begin{array}{c}\text { Polymorphism } \\
\text { information content }\end{array}$ \\
\hline Aa02944 & 0.46 & 8 & 0.71 & 0.68 \\
Aa29706 & 0.31 & 9 & 0.78 & 0.75 \\
Aa31542 & 0.52 & 9 & 0.67 & 0.63 \\
Aa22416 & 0.24 & 13 & 0.87 & 0.85 \\
Aa23178 & 0.28 & 14 & 0.86 & 0.85 \\
Aa24810 & 0.42 & 10 & 0.75 & 0.72 \\
Aa18854 & 0.37 & 15 & 0.81 & 0.80 \\
Aa13813 & 0.34 & 9 & 0.76 & 0.72 \\
Aa24840 & 0.54 & 7 & 0.66 & 0.64 \\
Aa32255 & 0.34 & 11 & 0.75 & 0.72 \\
Aa23930 & 0.62 & 6 & 0.56 & 0.53 \\
Aa01237 & 0.43 & 8 & 0.73 & 0.70 \\
Aa26466 & 0.50 & 8 & 0.66 & 0.62 \\
Aa38820 & 0.33 & 13 & 0.77 & 0.74 \\
Aa02342 & 0.50 & 7 & 0.67 & 0.62 \\
Aa00689 & 0.33 & 6 & 0.72 & 0.67 \\
Aa03927 & 0.27 & 12 & 0.84 & 0.82 \\
Aa16574 & 0.61 & 6 & 0.57 & 0.52 \\
Mean & 0.41 & 9.5 & 0.73 & 0.70 \\
\hline
\end{tabular}

same location or a different location, whereas six populations held at minimum two clones from the same collection location. Clonal genotypes from identical collection sites were used to create the clone-corrected data set, in which all but one genotype per MLG was eliminated for each collection site. A total of 46 clonal genotypes accounted for 14 MLGs across the six consensus populations (Table 2), indicating that the clonal genotypes were spread relatively uniformly among these MLGs. The $P_{\text {sex }}$ values for each of the 14 clonal MLGs were significantly low; thus, each of the clonal MLGs was likely the result of spread via mycelium within the interconnected canopy of the same tree or an effect of the homothallic nature of A. anomala and corresponded to the collection of samples from related infection events or lineages.

The number of individuals per population in the full data set ranged from 7 to 36 . In the clone-corrected data set (described prior) the number of individuals per population ranged from 3 to 36 . In both the full and clone-corrected data sets, the total number of MLGs ranged from 3 to 35 per population across all 11 consensus populations. The frequency of the most common genotype in the complete data set was 7 but fell to 2 in the clone-corrected data set. The lowest frequency of the most common genotype in any population across both data sets was 1 . The multilocus genotypic diversity (probability that two random accessions in a population will have different genotypes) ranged from 0.6 to 1 across populations in the complete data set and from 0.95 to 1 across populations for the clone-corrected data set. The genotypic diversity of the entire complete data set was 0.99 , whereas the genotypic diversity of the entire clone-corrected data set was 1.0.

Multilocus linkage disequilibrium was assessed for both data sets based on the index of association $I_{A}$ and the modified version of $I_{A}, \bar{r}_{d}$ (Table 2). Both the complete and clone-corrected data sets resulted in significant linkage disequilibrium (clonal reproduction) as shown by the $I_{A}$ and $\bar{r}_{d}$ values at $P \leq 0.05$ for the following populations: $1,2,6,7,9,10$, and 11 . The remaining four populations resulted in $I_{A}$ and $\bar{r}_{d}$ values that were not significantly different from zero and thus were not considered to be in linkage disequilibrium.

AMOVA analysis. An AMOVA was performed on both the full and clone-corrected data sets (Table 3). Both AMOVAs were analyzed based on the DAPC-derived populations, in which the clone-corrected data set held fewer genotypes in six of the populations. In total, 11 populations were analyzed to determine whether they were statistically distinct populations. In both AMOVA outputs, the pairwise $\Phi_{\mathrm{PT}}$ statistics indicated that each

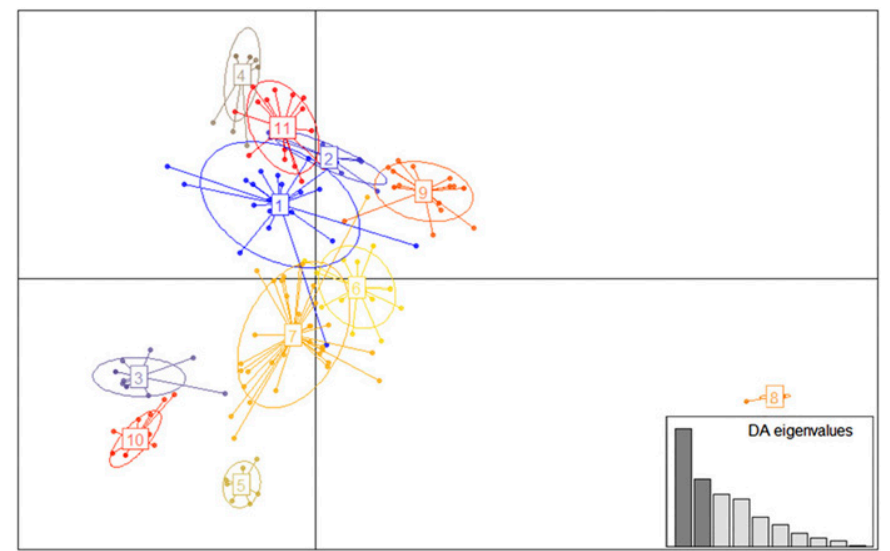

Fig. 2. Scatterplot from a discriminant analysis of principal components of the first two principal components resolved using the software program $\mathrm{R}$ ( $\mathrm{R}$ Core Team 2018) with the package Adegenet (Jombart 2008; Jombart and Ahmed 2011). Eleven clusters of accessions were assigned based on the Bayesian information criterion and are each represented as an inertia ellipse. DA = discriminant analysis. 
of the 11 populations was statistically distinct $(P \leq 0.01)$. This result illustrates that there are at least 11 statistically supported genetic populations of $A$. anomala present in the study.

The partitioned population variance was also resolved by the AMOVA. A total of $51 \%$ of the variance was apportioned among populations and the remaining $49 \%$ was within populations. Note that this variance was shifted slightly for the clone-corrected data set, in which interpopulation variance was $57 \%$ and intrapopulation variance $43 \%$. Interpopulation variance was further partitioned into each of the 11 populations and was variable across groups, $0.74 \%$

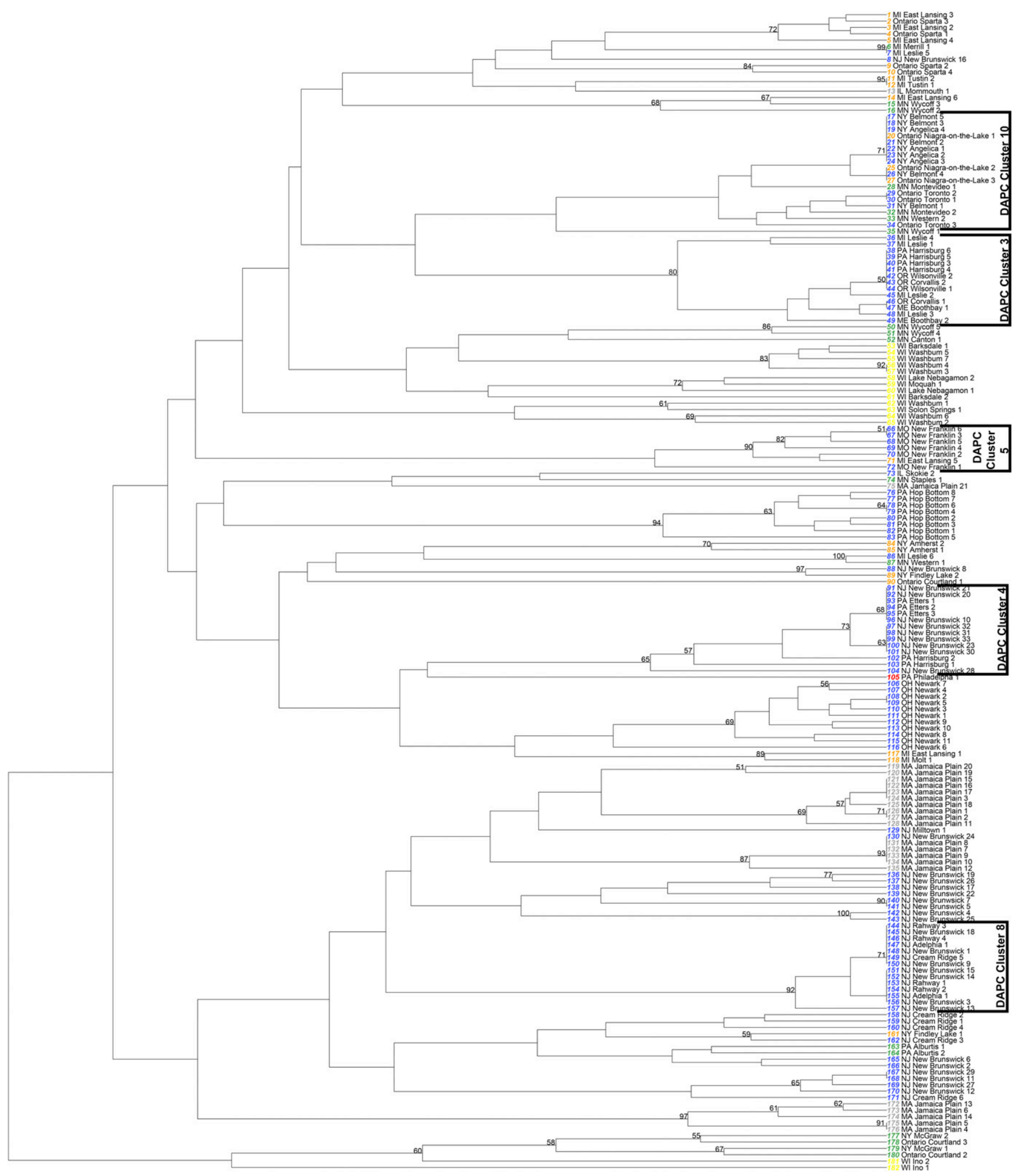

Fig. 3. Using 18 primers for each of the 182 accessions, a unweighted pair group method with arithmetic mean dendrogram was prepared with R software (R Core Team 2018) using Bruvo's genetic distance in the R package poppr version 2.8.0 (Kamvar et al. 2014, 2015). Bootstrap support values derived from 1,000 replicates are included at the dendrogram nodes. Colored accession identification numbers indicate the host species that each accession was collected from: blue from Corylus avellana, yellow from C. americana, orange from a Corylus hybrid, red from C. colurna, and gray from unknown Corylus species. DAPC = discriminant analysis of principal components. 
(population 8) versus $33.69 \%$ (population 7). Similarly, the variance across the populations ranged from $0.24 \%$ (population 8 ) to $33.69 \%$ (population 7) for both the complete and clone-corrected data sets (Table 3).

Spatial autocorrelation. A correlogram (Fig. 4) was used to discern the geographic distances (distance classes) at which the genetic population structure of $A$. anomala was statistically correlated to collection location. The extent of genetic structure, or patch size at which the intrapopulation structure was found, was the distance class of 33 . Thus, accessions collected within $33 \mathrm{~km}$ of each other tended to have similar or closely related genotypes. In addition, oscillation of the autocorrelation coefficient $(r)$ above and below 0 was observed through the distance class of $\sim 400 \mathrm{~km}$, and significantly positive correlation between genotypes and location was found at distance classes 224 and 396, which is indicative of microspatial structure. This microspatial structure implies that there is patchiness (clustering) of accessions with similar genotypes, where (beyond $33 \mathrm{~km}$ ) small clusters of accessions with similar genotypes tended to aggregate at distances of 224 and $396 \mathrm{~km}$ apart.

\section{DISCUSSION}

A total of 11 genetic populations were identified through the DAPC scatterplot. The dendrogram topology and DAPC analysis were then compared, and five populations were fully corroborated. The 11 DAPC populations were statistically supported by the AMOVA. Further individual and MLG analysis of the population structure of our collection of 182 accessions corroborated the high level of genetic diversity illustrated in both the UPGMA cluster and the DAPC analysis. Overall, these results support earlier work showing that $A$. anomala is genetically diverse (Cai et al. 2013). Additionally, because of the scope of the sampling range of this study, we were able to resolve clustering of many highly similar genotypes at a regional level.

TABLE 2. Multilocus genotype (MLG) and linkage disequilibrium analysis results for each of the 11 populations, and full data sets with and without clonal genotypes

\begin{tabular}{|c|c|c|c|c|c|c|c|}
\hline Population $^{\mathrm{a}}$ & $\begin{array}{l}\text { Number of } \\
\text { accessions }\end{array}$ & $\begin{array}{l}\text { Number of } \\
\text { MLGs }(g)\end{array}$ & $\begin{array}{l}\text { Frequency of the } \\
\text { most common MLG }\end{array}$ & $\hat{\mathrm{G}}^{\mathrm{b}}$ & $I_{A}{ }^{\mathrm{c}}$ & $\bar{r}_{d}^{\mathrm{d}}$ & Accessions with repeated MLGs \\
\hline 1 & 20 & 19 & 2 & 0.99 & $1.11 * * *$ & $0.07 * * *$ & \\
\hline 2 & 8 & 8 & 1 & 1.00 & $3.61 * * *$ & $0.28 * * *$ & \\
\hline 3 & 14 & 9 & 3 & 0.91 & $0.90^{\mathrm{ns}}$ & $0.13 * * *$ & $\begin{array}{l}\text { PA_Harrisburg_4, PA_Harrisburg_6, } \\
\text { PA_Harrisburg_3, OR_Wilsonville_1, } \\
\text { OR_Wilsonville_2, OR_Corvallis_2 }\end{array}$ \\
\hline $3^{\mathrm{nc}}$ & 10 & 9 & 2 & 0.96 & $0.65^{\mathrm{ns}}$ & $0.10 *$ & \\
\hline 4 & 14 & 6 & 5 & 0.81 & $1.14^{\mathrm{ns}}$ & $0.17 * *$ & $\begin{array}{l}\text { NJ_NewBrunswick_23, } \\
\text { NJ_NewBrunswick_31, } \\
\text { NJ_NewBrunswick_30, } \\
\text { NJ_NewBrunswick_32, } \\
\text { NJ_NewBrunswick_33, PA_Etters_1, } \\
\text { PA_Etters_3, NJ_NewBrunswick_10, } \\
\text { NJ_NewBrunswick_20, } \\
\text { NJ_NewBrunswick_21 }\end{array}$ \\
\hline $4^{\mathrm{nc}}$ & 7 & 6 & 2 & 0.95 & $1.00^{\mathrm{ns}}$ & $0.14 *$ & \\
\hline 5 & 7 & 6 & 2 & 0.95 & $0.86^{\mathrm{ns}}$ & $0.21 *$ & \\
\hline 6 & 16 & 15 & 2 & 0.99 & $2.82 * * *$ & $0.16^{* * *}$ & PA_HopBottom_4, PA_HopBottom_6 \\
\hline $6^{\mathrm{nc}}$ & 15 & 15 & 1 & 1.00 & $2.56 * * *$ & $0.15^{* * *}$ & \\
\hline 7 & 36 & 35 & 2 & 1.00 & $1.66 * * *$ & $0.11 * * *$ & \\
\hline 8 & 14 & 3 & 7 & 0.60 & $0^{\text {ns }}$ & $0^{\text {ns }}$ & $\begin{array}{l}\text { NJ_NewBrunswick_14, } \\
\text { NJ_NewBrunswick_34, } \\
\text { NJ_NewBrunswick_15, } \\
\text { NJ_NewBrunswick_3, NJ_Rahway_1, } \\
\text { NJ_Rahway_2, NJ_Rahway_4, } \\
\text { NJ_NewBrunswick_9, } \\
\text { NJ_NewBrunswick_35, } \\
\text { NJ_NewBrunswick_18, } \\
\text { NJ_NewBrunswick_1, NJ_Rahway_3, } \\
\text { NJ_CreamRidge_5 }\end{array}$ \\
\hline $8^{\mathrm{nc}}$ & 3 & 3 & 1 & 1.00 & $-0.50^{\mathrm{ns}}$ & $-0.50^{\mathrm{ns}}$ & \\
\hline 9 & 19 & 15 & 3 & 0.97 & $1.92 * * *$ & $0.22 * * *$ & $\begin{array}{l}\text { NJ_NewBrunswick_24, } \\
\text { MA_JamaicaPlain_10, } \\
\text { MA_JamaicaPlain_15, } \\
\text { MA_JamaicaPlain_17, } \\
\text { MA_JamaicaPlain_9, } \\
\text { MA_JamaicaPlain_10 }\end{array}$ \\
\hline $9^{\text {ne }}$ & 16 & 15 & 2 & 1.00 & $1.56 * * *$ & $0.18 * * *$ & \\
\hline 10 & 18 & 10 & 6 & 0.88 & $1.26 * * *$ & $0.19 * * *$ & $\begin{array}{l}\text { NY_Belmont_5, NY_Belmont_3, } \\
\text { Ontario_Niagraonthelake_1, } \\
\text { NY_Angelica_3, NY_Angelica_1, } \\
\text { NY_Angelica_4, NY_Angelica_2, } \\
\text { Ontario_Niagraonthelake_2, } \\
\text { Ontario_Niagraonthelake_3 }\end{array}$ \\
\hline $10^{\mathrm{nc}}$ & 12 & 10 & 2 & 0.97 & $0.72 * * *$ & $0.11 * * *$ & \\
\hline 11 & 16 & 15 & 2 & 0.98 & $2.35 * * *$ & $0.17 * * *$ & \\
\hline Total population & 182 & 141 & 7 & 0.99 & 2.08 & 0.12 & \\
\hline Total population ${ }^{\mathrm{nc}}$ & 144 & 135 & 2 & 1.00 & $1.64 * * *$ & $0.10 * * *$ & \\
\hline
\end{tabular}

a The "nc" superscript indicates populations where all but one clonal genotype per location was taken out.

b $\hat{\mathrm{G}}$ is the probability that two random individuals in a population have a different MLG.

c $I_{A}$ is the index of association, where $P$ values are $* P \leq 0.05$, ** $P \leq 0.01$, and $* * * P \leq 0.001$. The "ns" superscript indicates not significant.

$\mathrm{d} \bar{r}_{d}$ is a modified $I_{A}$ statistic, where the value is independent of the number of loci analyzed. *P $\leq 0.05, * * P \leq 0.01$, and $* * * P \leq 0.001$. 
Based on the SSR marker statistics, the A. anomala accessions sampled in our study were found to be genetically diverse in comparison with other fungal diversity studies using a similar approach and marker system. For example, the mean number of alleles per locus was 5.2 for Metarhizium anisopliae var. anisopliae (Becerra Velásquez et al. 2007), 7.9 for Pyricularia oryzae (Adreit et al. 2007), and 9.3 for Beauveria bassiana (Wang et al. 2005), all of which were lower than the average number of alleles per locus for A. anomala (9.5). Furthermore, the mean gene diversity value of A. anomala $(0.7308)$ was higher than that of P. oryzae $(0.447)$ (Adreit et al. 2007).

In addition to locus-level genetic diversity, MLG statistics were also indicative of genotype diversity. Of the 141 MLGs identified, 138 were represented by a single, unique genotype, thus $>75 \%$ of the accessions included in this study were considered to be genetically distinct. The remaining $25 \%$ of the (clonal) genotypes were evenly dispersed across over half of the DAPC populations. The frequency of the most common genotype in the data set was 7; thus, the greatest representation of one MLG in the entire population (data set) was $<4 \%$. In addition, genotypic diversity values of the full and clone-corrected data set were nearly 1 . High proportions of unique MLGs and low representation of any one genotype further corroborate the presence of high genetic diversity within A. anomala. Other researchers have shown that high genotypic diversity is not uncommon for a homothallic fungus. Reproductive modes of fungi (asexual or sexual) are not necessarily linked to one single reproductive strategy (homothallism or heterothallism) (Taylor et al. 1999). Recombinant genotypes could be attributable to high marker mutation rates, which is particularly true of SSR markers ( $\mathrm{Li}$ et al. 2002; Lynch et al. 2008). Given the exceptionally large genome size of $A$. anomala $(>300 \mathrm{Mb})$ and high transposable element content (>85\%) (B. Hillman, unpublished data), high rates of recombination may not be surprising.

Beyond the high genetic diversity resolved in this study, statistical analysis of the individual DAPC-derived populations suggests that relationships also exist between genotype and geographic origin. As previously stated, the DAPC scatterplot and UPGMA dendrogram helped to elucidate the number of populations this sample set of accessions grouped into based on their allelic profiles. For eight of the groups, specimens collected from one geographic location comprised $\geq 50 \%$ of the total. Thus, samples collected from the same location tended to cluster together based on their genotypes. The resulting AMOVA of the DAPC clusters showed that the variation among all 11 populations was moderate
(43 to 51\%; clone-corrected and complete data sets, respectively). In comparison, the sum of the diversity within each of these populations was half of the interpopulation diversity (49 to 57\%), although it was not evenly partitioned among all 11 populations (0.24 to $33.69 \%)$.

The autocorrelation analysis corroborated this finding, in that it showed that samples collected within certain proximities $(33,224$, and $396 \mathrm{~km}$ apart) are more genetically similar than those found further than $443 \mathrm{~km}$ apart. The steep peaks and oscillation of the $r c$ values of the correlogram are indicative of microspatial genetic structure, where high-density populations of similar genotypes are separated by intervening gaps of low densities of similar genotypes (Peakall et al. 2003; Smouse and Peakall 1999; Sokal and Wartenberg 1983).

The $I_{A}$ and $\bar{r}_{d}$ values both lent further insight into the genetic relationships of accessions placed within each population. The majority of the populations (seven of 11) resulted in significantly high $I_{A}$ and $\bar{r}_{d}$ values, which illustrates that those populations were in linkage disequilibrium and consistent with homothallism (Cai et al. 2013), indicative of nonrandom mating (Milgroom et al. 2009). Of the seven populations containing a large proportion of accessions with the same genotype, the majority of the accessions in each of the groups were collected from single locations.

The regional genotypic specificity exhibited though the population structure of $A$. anomala supports anecdotal evidence of movement and proliferation of $A$. anomala throughout the collection sites in North America. One example can be visualized in Figure 3, which depicts DAPC cluster 3 with 14 accessions, four of which constituted all of the accessions collected in Oregon (two

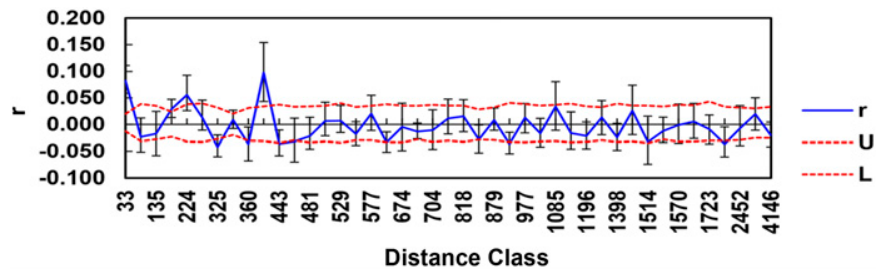

Fig. 4. A spatial correlogram depicting the correlation between the genetic and physical distance between accessions. The degree of correlation is expressed as the correlation coefficient, which is denoted as $(r)$ on the vertical axis. The upper and lower boundaries of statistical significance at the $P<0.001$ level are denoted by the boundary lines $U$ and $L$, respectively.

TABLE 3. Apportionment of total within-population variance into 11 populations, with and without clonal genotypes

\begin{tabular}{|c|c|c|c|c|c|}
\hline Population $^{\mathrm{a}}$ & Number & Degrees of freedom & Sum of squares within populations ${ }^{b}$ & Variance components within populations ${ }^{c}$ & Proportion of total variance ${ }^{\mathrm{d}}$ \\
\hline 1 & 20 & 19 & 91.40 & 4.81 & 15.19 \\
\hline 2 & 8 & 7 & 28.63 & 4.09 & 4.76 \\
\hline 3 & 14 & 13 & 23.79 & 1.83 & 3.95 \\
\hline $3^{\text {nc }}$ & 10 & 9 & 19.30 & 2.14 & 3.52 \\
\hline 4 & 14 & 13 & 18.36 & 1.41 & 3.05 \\
\hline $4^{\mathrm{nc}}$ & 7 & 6 & 14.14 & 2.36 & 2.58 \\
\hline 5 & 7 & 6 & 9.43 & 1.57 & 1.57 \\
\hline 6 & 16 & 15 & 82.31 & 5.49 & 13.68 \\
\hline $6^{\mathrm{nc}}$ & 15 & 14 & 79.47 & 5.68 & 14.49 \\
\hline 7 & 36 & 35 & 202.67 & 5.79 & 33.69 \\
\hline 8 & 14 & 13 & 4.43 & 0.34 & 0.74 \\
\hline $8^{\text {nc }}$ & 3 & 2 & 1.33 & 0.67 & 0.24 \\
\hline 9 & 19 & 18 & 56.42 & 3.14 & 9.38 \\
\hline $9^{\text {nc }}$ & 16 & 15 & 48.56 & 3.24 & 8.85 \\
\hline 10 & 18 & 17 & 29.67 & 1.75 & 4.93 \\
\hline $10^{\mathrm{nc}}$ & 12 & 11 & 23.00 & 2.10 & 4.19 \\
\hline 11 & 16 & 15 & 54.50 & 3.63 & 9.06 \\
\hline
\end{tabular}

a The "nc" superscript indicates populations where all but one clonal genotype per location was taken out.

b Sum of squares within populations quantifies the variation among accessions within each population.

c Variance components within populations are calculated by dividing the sum of squares within populations by the degrees of freedom in that population.

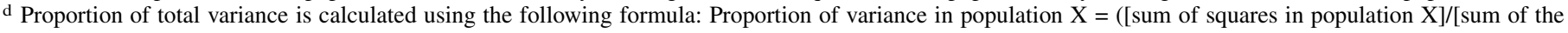
sum of squares in each population] $) \times 100$. 
from Corvallis and two from Wilsonville). This location is of particular interest because it is a place where the fungus is not endemic and was introduced in the 1960s from an unknown source (Davison and Davidson 1973). There is significant bootstrap support for this grouping (0.8), as well as a significantly high $I_{A}$ value indicating a significant rate of linkage disequilibrium. Although the two sample locations in Oregon (Corvallis and Wilsonville) are over $100 \mathrm{~km}$ apart, both are represented by nearly identical genotypes, which supports previous studies suggesting that infection in the Pacific Northwest originated from a single point introduction (Gottwald and Cameron 1980b; Pinkerton et al. 1992). However, additional samples are needed to substantiate this claim, which is work that is currently being completed by the authors. Other accessions included in this cluster were collected from Boothbay, Maine, Leslie, Michigan, and Harrisburg, Pennsylvania. These accessions, which were shown to have very similar genotypes but were collected from disparate locations, may be indicative of movement of the fungus by humans to or from Oregon, likely through shipments of nursery stock harboring latent infections. As further support, two of the collection sites have records of ordering hazelnut plant material from the same mail-order nursery located in Oregon.

The results of this study expand knowledge on the diversity and population structure of $A$. anomala, the causal agent of EFB, a disease that is considered to be the greatest limiting factor to hazelnut production in North America. In summary, A. anomala appears to be very genetically diverse with a population structure showing genotype collection location specificity on a regional and local scale. Interestingly, there was no significant correlation between the accession host and accession genotype. These findings can have important implications for managing disease in hazelnut orchards and when attempting to breed hazelnut plants resistant to EFB. For example, two public hazelnut breeding programs in the United States (OSU and Rutgers University) have goals of developing plants that express high, durable resistance to this disease (Mehlenbacher 1994; Molnar et al. 2005). In our study, the samples collected from Oregon fell into only one genetic population, indicating that trees grown in that region may be exposed to only a limited pool of $A$. anomala genotypes. These findings support the previous reports of a single point of entry to the region (Gottwald and Cameron 1980b). If existing quarantine measures are maintained in the Pacific Northwest where movement of Corylus spp. plant material is restricted from the East (Barss 1930), the limited genotype diversity in the region may remain and possibly support longevity of the single Gasaway $R$-gene currently being used to protect plants in the region (Mehlenbacher et al. 1991). However, as a result, breeders in the region remain limited to working with only a small subset of the fungal diversity, possibly limiting their ability to select plants expressing broader resistance (Capik and Molnar 2012; Molnar et al. 2010a, 2010b).

In contrast to the scenario in Oregon, samples collected in New Brunswick, New Jersey, fell into seven distinct populations, suggesting that trees grown in this region are likely to encounter a greater diversity of A. anomala genotypes. One of these, DAPC population 8 , was the most strongly differentiated of the 11 total populations. This wider diversity may present a more challenging scenario for managing EFB in orchards, many of which are expected to last $>35$ years, as well as identifying and/or breeding trees that express long-lived resistance to EFB. Thus, a breeding approach bolstered by the knowledge that $A$. anomala appears to be a very diverse organism with some regional population structure and indication of proliferation of genetically distinct populations (population 8) should be considered to help develop plants expressing durable forms of resistance. Single gene resistance should be vetted thoroughly across regions (and/or through controlled exposure to known accessions), with multigenic (quantitative) sources of resistance including $C$. americana, $C$. avellana, and $R$-gene pyramiding explored and considered as part of long-term breeding objectives. Additional breeding strategies should include strengthened regional collaboration among public and private breeding programs to share germplasm and assess resistance to the pathogen across a wide diversity of accessions and genetic backgrounds of the host plants.

\section{ACKNOWLEDGMENTS}

We thank J. Capik for sample collection, C. Leadbetter for collection and microsatellite marker screening, and G. Cai for microsatellite marker design.

\section{LITERATURE CITED}

Adreit, H., Santoso, D., Andriantsimialona, D., Utami, D. W., Notteghem, J. L., Lebrun, M. H., and Tharreau, D. 2007. Microsatellite markers for population studies of the rice blast fungus, Magnaporthe grisea. Mol. Ecol. Notes 7:667-670.

Agapow, P. M., and Burt, A. 2001. Indices of multilocus linkage disequilibrium. Mol. Ecol. Notes 1:101-102.

Barss, H. P. 1921. The eastern filbert blight menace. Proc. West. Nut Growers Assoc. 4:31-33.

Barss, H. P. 1930. Eastern filbert blight. Calif. Agric. Dep. Bull. 19:489-490.

Becerra Velásquez, V., Cárcamo, M. P., Meriño, C. R., Inglesias, A. F., and Durán, J. F. 2007. Intraspecific differentiation of Chilean isolates of the entomopathogenic fungi Metarhizium anisopliae var. anisopliae as revealed by RAPD, SSR and ITS markers. Genet. Mol. Biol. 30:89-99.

Brownstein, M. J., Carpten, J. D., and Smith, J. R. 1996. Modulation of nontemplated nucleotide addition by Taq DNA polymerase: Primer modifications that facilitate genotyping. BioTechniques 20:1004-1010.

Cai, G., Leadbetter, C. W., Muehlbauer, M. F., Molnar, T. J., and Hillman, B. I. 2013. Genome-wide microsatellite identification in the fungus Anisogramma anomala using Illumina sequencing and genome assembly. PLoS One 8:e82408.

Capik, J. M., and Molnar, T. J. 2012. Assessment of host (Corylus sp.) resistance to eastern filbert blight in New Jersey. J. Am. Soc. Hortic. 137:157-172.

Capik, J. M., Muehlbauer, M., Novy, A., Honig, J. A., and Molnar, T. J. 2013. Eastern filbert blight-resistant hazelnuts from Russia, Ukraine, and Poland. HortScience 48:466-473.

Chen, H., Mehlenbacher, S. A., and Smith, D. C. 2005. AFLP markers linked to eastern filbert blight resistance from OSU 408.040 hazelnut. J. Am. Soc. Hortic. Sci. 130:412-417.

Chen, H., Mehlenbacher, S. A., and Smith, D. C. 2007. Hazelnut accessions provide new sources of resistance to eastern filbert blight. HortScience 42: 466-496.

Colburn, B. C., Mehlenbacher, S. A., Sathuvalli, V. R., and Smith, D. C. 2015. Eastern filbert blight resistance in hazelnut accessions 'Culplà', Crvenje', and OSU 495.072. J. Am. Soc. Hortic. Sci. 140:191-200.

Coyne, C. J., Mehlenbacher, S. A., and Smith, D. C. 1998. Sources of resistance to eastern filbert blight. J. Am. Hortic. Sci. 124:253-257.

Davison, A. D., and Davidson, R. M. 1973. Apioporthe and Monchaetia canker reported in western Washington. Plant Dis. Rep. 57:522-523.

Ellis, J. B., and Everhart, B. M. 1892. The North American Pyrenomycetes. Ellis and Everhart, Newfield, NJ.

European and Mediterranean Plant Protection Organization. 2012. EPPO A1 list of pests recommended for regulation as quarantine pests. https:// www.eppo.int/QUARANTINE/listA1.htm

Fuller, A. S. 1908. The Nut Culturist. Orange Judd, New York.

Gottwald, T., and Cameron, H. 1980a. Infection site, infection period, and latent period of canker caused by Anisogramma anomala in European filbert. Phytopathology 70:1083-1087.

Gottwald, T., and Cameron, H. 1980b. Disease increase and the dynamics of spread of canker caused by Anisogramma anomala in European filbert in the Pacific Northwest. Phytopathology 70:1087-1092.

Gottwald, T. R., and Cameron, H. R. 1979. Morphology and life history of Anisogramma anomala. Mycologia 71:1107-1126.

Johnson, K. B., and Pinkerton, J. N. 2002. Eastern filbert blight. Pages 44-46 in: Compendium of Nut Crop Diseases in Temperate Zones. B. L. Teviotdale, T. J. Michailides, and J. W. Pscheidt, eds. American Phytopathological Society, St. Paul, MN.

Jombart, T. 2008. adegenet: A R package for the multivariate analysis of genetic markers. Bioinformatics 24:1403-1405.

Jombart, T., and Ahmed, I. 2011. adegenet 1.3-1: New tools for the analysis of genome-wide SNP data. Bioinformatics 27:3070-3071.

Julian, J., Seavert, C., and Olsen, J. L. 2009. An economic evaluation of the impact of eastern filbert blight resistant cultivars in Oregon, U.S.A. Acta Hortic.: 725-732. 
Kamvar, Z. N., Brooks, J. C., and Grünwald, N. J. 2015. Novel R tools for analysis of genome-wide population genetic data with emphasis on clonality. Front. Genet. 6:208.

Kamvar, Z. N., Tabima, J. F., and Grünwald, N. J. 2014. Poppr: An R package for genetic analysis of populations with clonal, partially clonal, and/or sexual reproduction. PeerJ 2:e281.

Leadbetter, C. W., Capik, J. M., Mehlenbacher, S. A., and Molnar, T. J. 2016. Hazelnut accessions from Russia and Crimea transmit resistance to eastern filbert blight. J. Am. Pomol. Soc. 70:92-109.

Leadbetter, C. W., Capik, J. M., Pisetta, M., and Molnar, T. J. 2015. Sources of resistance to eastern filbert blight in hazelnuts from the Republic of Georgia. Sci. Hortic. (Amsterdam) 193:269-275.

Li, Y. C., Korol, A. B., Fahima, T., Beiles, A., and Nevo, E. 2002. Microsatellites: Genomic distribution, putative functions and mutational mechanisms: A review. Mol. Ecol. 11:2453-2465.

Liu, K., and Muse, S. V. 2005. PowerMarker: An integrated analysis environment for genetic marker analysis. Bioinformatics 21:2128-2129.

Lunde, C. F., Mehlenbacher, S. A., and Smith, D. C. 2000. Survey of hazelnut cultivars for response to eastern filbert blight inoculation. HortScience 35:729-731.

Lynch, M., Sung, W., Morris, K., Coffey, N., Landry, C. R., Dopman, E. B., Dickinson, W. J., Okamoto, K., Kulkarni, S., Hartl, D. L., and Thomas, W. K. 2008. A genome-wide view of the spectrum of spontaneous mutations in yeast. Proc. Natl. Acad. Sci. 105:9272-9277.

Mehlenbacher, S. A. 1994. Genetic improvement of the hazelnut. Acta Hortic.: 23-38

Mehlenbacher, S. A., Brown, R. N., Davis, J. W., Chen, H., Bassil, N. V., Smith, D. C., and Kubisiak, T. L. 2004. RAPD markers linked to eastern filbert blight resistance in Corylus avellana. Theor. Appl. Genet. 108:651-656.

Mehlenbacher, S. A., Smith, D. C., and McCluskey, R. L. 2009. 'Yamhill' hazelnut. HortScience 44:845-847.

Mehlenbacher, S. A., Smith, D. C., and McCluskey, R. L. 2011. 'Jefferson' hazelnut. HortScience 46:662-664.

Mehlenbacher, S. A., Smith, D. C., and McCluskey, R. L. 2013. 'Dorris' hazelnut. HortScience 48:796-799.

Mehlenbacher, S. A., Smith, D. C., and McCluskey, R. L. 2014. 'Wepster' hazelnut. HortScience 49:346-349.

Mehlenbacher, S. A., Thompson, M. M., and Cameron, H. R. 1991. Occurrence and inheritance of resistance to eastern filbert blight in 'Gasaway' hazelnut. HortScience 26:410-411.

Milgroom, M. G., Sotirovski, K., Risteski, M., and Brewer, M. T. 2009. Heterokaryons and parasexual recombinants of Cryphonectria parasitica in two clonal populations in southeastern Europe. Fungal Genet. Biol. 46:849-854.

Molnar, T. J., Capik, J., Zhao, S., and Zheng, N. 2010a. First report of eastern filbert blight on Corylus avellana 'Gasaway' and 'VR 20-11' caused by Anisogramma anomala in New Jersey. Plant Dis. 94:1265.

Molnar, T. J., Goffreda, J. C., and Funk, C. R. 2005. Developing hazelnuts for the eastern United States. Acta Hortic.: 609-618.

Molnar, T. J., Goffreda, J. C., and Funk, C. R. 2010b. Survey of Corylus resistance to Anisogramma anomala from different geographic locations. HortScience 45:832-836.

Molnar, T. J., Mehlenbacher, S. A., Zaurov, D. E., and Goffreda, J. C. 2007. Survey of hazelnut germplasm from Russia and Crimea for response to eastern filbert blight. HortScience 42:51-56.

Muehlbauer, M. F., Honig, J. A., Capik, J. M., Vaiciunas, J. N., and Molnar, T. J. 2014. Characterization of eastern filbert blight-resistant hazelnut germplasm using microsatellite markers. J. Am. Hortic. Sci. 139:399-432.

Osterbauer, N. K. 1996. Genetic Variability in the Eastern Filbert Blight Pathosystem. Ph.D. dissertation, Department of Horticulture, Oregon State University, Corvallis.

Peakall, R., Ruibal, M., and Lindenmayer, D. B. 2003. Spatial autocorrelation analysis offers new insights into gene flow in the Australian bush rat, Rattus fuscipes. Evolution 57:1182-1195.
Peakall, R., and Smouse, P. E. 2012. GenAlEx 6.5: Genetic analysis in Excel. Population genetic software for teaching and research-an update. Bioinformatics 28:2537-2539.

Peakall, R. O. D., and Smouse, P. E. 2006. GENALEX 6: Genetic analysis in excel, population genetic software for teaching and research. Mol. Ecol. Notes 6:288-295.

Peck, C. H. 1874. Report of the botanist. Ann. Rep. N. Y. State Mus. Nat. Hist. 28:27.

Pinkerton, J., Johnson, K., Stone, J., and Ivors, K. 1998. Factors affecting the release of ascospores of Anisogramma anomala. Phytopathology 88: $122-128$

Pinkerton, J., Johnson, K., Theiling, K., and Griesbach, J. 1992. Distribution and characteristics of the eastern filbert blight epidemic in western Oregon. Plant Dis. 76:1179-1182.

Pinkerton, J. N., Johnson, K. B., Mehlenbacher, S. A., and Pscheidt, J. W. 1993. Susceptibility of European hazelnut clones to eastern filbert blight. Plant Dis. 77:261-266.

Pinkerton, J. N., Stone, J. K., Nelson, S. J., and Johnson, K. B. 1995. Infection of European hazelnut by Anisogramma anomala: Ascospore adhesion, mode of penetration of immature shoots, and host response. Phytopathology $85: 1260-1268$

Pscheidt, J., Grimaldi, P., and Penhallegon, R. 2014. Eastern filbert blight epidemic in the Pacific Northwest. Oregon State University. http:/oregonstate.edu/dept/botany/epp/EFB/location/map1.htm

R Core Team. 2018. R: A language and environment for statistical computing. R Foundation for Statistical Computing, Vienna, Austria. https://www.Rproject.org/

Sathuvalli, V., Chen, H. L., Mehlenbacher, S. A., and Smith, D. C. 2011a. DNA markers linked to eastern filbert blight resistance in 'Ratoli' hazelnut. Tree Genet. Genomes 7:337-345.

Sathuvalli, V., Mehlenbacher, S. A., and Smith, D. C. 2009. New sources of eastern filbert blight and linked markers. Acta Hortic.: 123-126.

Sathuvalli, V., Mehlenbacher, S. A., and Smith, D. C. 2010. Response of hazelnut accessions to greenhouse inoculation with Anisogramma anomala. HortScience 45:1116-1119.

Sathuvalli, V., Mehlenbacher, S. A., and Smith, D. C. 2011b. DNA markers linked to eastern filbert blight resistance from a hazelnut selection from the Republic of Georgia. J. Am. Soc. Hortic. Sci. 136:350-357.

Schuelke, M. 2000. An economic method for the fluorescent labeling of PCR fragments. Nat. Biotechnol. 18:233-234.

Smouse, P. E., and Peakall, R. 1999. Spatial autocorrelation analysis of individual multiallele and multilocus genetic structure. Heredity 82:561573.

Sokal, R. R., and Wartenberg, D. E. 1983. A test of spatial autocorrelation analysis using an isolation-by-distance model. Genetics 105:219-237.

Stenberg, P., Lundmark, M., and Saura, A. 2003. MLGsim: A program for detecting clones using a simulation approach. Mol. Ecol. Notes 3:329331.

Stone, J., Pinkerton, J., and Johnson, K. 1994. Axenic culture of Anisogramma anomala: Evidence for self-inhibition of ascospore germination and colony growth. Mycologia 86:674-683.

Taylor, J. W., Jacobson, D. J., and Fisher, M. C. 1999. The evolution of asexual fungi: Reproduction, speciation and classification. Annu. Rev. Phytopathol. 37:197-246.

Thompson, M. M., Lagerstedt, H. B., and Mehlenbacher, S. A. 1996. Hazelnuts. Pages 125-184 in: Fruit Breeding, Vol. 3. Nuts. J. Janik and J. N. Moore, eds. Wiley, New York.

Wang, S., Miao, X., Zhao, W., Huang, B., Fan, M., Li, Z., and Huang, Y. 2005. Genetic diversity and population structure among strains of the entomopathogenic fungus, Beauveria bassiana, as revealed by inter-simple sequence repeats (ISSR). Mycol. Res. 109:1364-1372.

Weschcke, C. 1954. Growing Nuts in the North. Webb, St. Paul, MN. 